\author{
JORGE J. GUMUCIO, EDITOR \\ Department of Internal Medicine \\ Division of Gastroenterology (111D) \\ VA Medical Center/University of Michigan \\ Ann Arbor, Michigan 48105
}

\section{Elsewhere Reviews \\ PHYSIOLOGICAL RESPONSES TO INCREASED DIETARY CHOLESTEROL: THE CASE OF THE EGG MAN}

Kern $F$ Jr. Normal plasma cholesterol in an 88-year-old man who eats 25 eggs a day. N Engl J Med 1991;324: 896-899.

\section{EDITOR'S SUMMARY}

An 88-yr-old man carrying the tentative diagnosis of Alzheimer's disease had a documented history of ingesting 20 to $30 \mathrm{eggs} / \mathrm{day}$ for approximately $15 \mathrm{yr}$. His psychiatrist characterized his eating habits as a compulsive disorder: "Eating these eggs ruins my life, but I can't help it." He is in excellent health. His weight is constant at 82 to $86 \mathrm{~kg}$ (height $1.87 \mathrm{~m}$ ). Apparently, he had a mild episode of angina. Otherwise, no significant history of stroke, heart disease or gallbladder disease was seen. His serum cholesterol levels have ranged between 150 and $200 \mathrm{mg} / \mathrm{dl}$.

\section{COMMENTS}

Individuals differ widely in their responses to dietary cholesterol. A rigorous cholesterol-lowering diet often can reduce serum cholesterol in hyperlipidemic patients by 40 to $50 \mathrm{mg} / \mathrm{dl}$ (1), and the feeding of high-cholesterol diets on a metabolic ward can increase mean serum cholesterol. However, many individuals exhibit little or no change in serum cholesterol despite large changes in diet. A broad division may be made between those individuals who, in response to changes in dietary cholesterol, display parallel changes in serum lipids (noncompensators) and those who do not (compensators). A recent study by McNamara and colleagues (2) found that of 75 male volunteers, $52(69 \%)$ were compensators, which was defined as a change of less than 5\% in plasma cholesterol after a dietary cholesterol challenge. The metabolic and genetic factors that determine our responses to dietary cholesterol are not well understood.

Kern in this unusual case has identified some novel mechanisms by which certain human beings may compensate for massive amounts of dietary cholesterol. The patient reported was an 88-yr-old man with a compulsive craving for eggs, which led him to consume 20 to 30 eggs (approximately 5,000 $\mathrm{mg}$ of cholesterol) each day for a period of at least $15 \mathrm{yr}$. Despite this practice, the patient (hereafter called "the egg man") had little evidence of atherosclerotic disease, and serum cholesterol measurements were consistently less than $200 \mathrm{mg} / \mathrm{dl}$. Using established methods, Kern measured cholesterol ab- sorption, cholesterol synthesis and bile acid kinetics and compared the results in this patient to findings in 11 normal individuals (controls) maintained for 2 to $3 \mathrm{wk}$ on low-cholesterol $(219 \mathrm{mg} /$ day) or high-cholesterol (1,156 mg/day) diets.

The metabolism of the egg man appears to have adapted in three ways to his massive cholesterol load. First, synthesis of cholesterol was $16 \%$ lower than in control subjects fed a low-cholesterol diet, a degree of suppression similar to that noted when control subjects consumed a high-cholesterol diet. Second, although total cholesterol absorption was high, fractional absorption of dietary cholesterol was only $18 \%$; this contrasted with mean cholesterol absorption of $55 \%$ and $46 \%$ for control subjects, respectively, on low-cholesterol or highcholesterol diets. Third, the egg man's rate of bile acid synthesis (the principal pathway of cholesterol degradation) was increased to levels twice those of controls on low-cholesterol diets; in contrast, the feeding of highcholesterol diets to control subjects produced no increase in bile acid synthesis. The bile acid pool size of the egg man also was twice a control's, indicating that his increased bile acid synthesis probably was not attributable to bile acid malabsorption.

The case of the egg man is important because it indicates that intestinal cholesterol absorption and hepatic bile acid synthesis in some human beings may be regulated over a wide range to compensate fully for changes in dietary cholesterol. Two important caveats are in order. Without restudying the same patient after a period of abstinence from cholesterol, we cannot be certain that the observed abnormalities are truly adaptive to dietary cholesterol, as opposed to unrelated genotypic or phenotypic variation. And even if the observed abnormalities are truly adaptive, we cannot estimate from this one case how frequently others would respond in the same way to such a massive cholesterol load. Nonetheless, it seems unlikely that the coexistence of three unusual findings (massive dietary cholesterol intake, low fractional cholesterol absorption and high bile acid synthesis) can be attributed to coincidence.

Absorption of cholesterol from the intestine is thought to be a passive process requiring solubilization with bile salts in mixed micelles and diffusion across an unstirred layer to the surface of the mucosal cell (3). The physical chemistry of this process is complex, and the specific rate-determining events are poorly understood. Animal studies suggest that the capacity for cholesterol uptake is limited (4), and it is possible that the low fractional absorption of cholesterol in Kern's patient results simply from saturation of a passive uptake system. 
Evidence suggests, however, that dietary cholesterol absorption may not be a completely passive phenomenon. Several authors have suggested the existence of a brush-border transport protein that may be involved in the uptake of cholesterol from the intestinal lumen (5, 6). Kern speculates that this putative transfer protein may be down-regulated in the egg man, leading to the unusually low fractional cholesterol absorption. One might further speculate that inhibitors directed against a cholesterol transfer protein would be useful hypocholesterolemic agents. Clearly, this is an area that requires further investigation.

The most intriguing finding in the egg man was his marked increase in bile acid synthesis-described by Kern as "greater than in any of the 200 subjects we have studied during the past 13 years." Bile acid synthesis is a major pathway of cholesterol elimination, and a mechanism linking the rate of bile acid synthesis to the cholesterol supply could contribute to cholesterol homeostasis. The role of cholesterol in regulation of human bile acid synthesis is controversial (7).

In the rat, activity of cholesterol $7 \alpha$-hydroxylase, the principal rate-determining enzyme in the bile acid biosynthetic pathway, increases in response to cholesterol feeding (8). Conversely, inhibition of cholesterol synthesis by inhibitors acting at early or late steps in the cholesterol biosynthetic pathway leads to rapid and reversible down-regulation of bile acid synthesis and cholesterol $7 \alpha$-hydroxylase activity $(9,10)$. Recent data indicate that these regulatory changes occur principally at a pretranslational level through effects on transcription of the cholesterol $7 \alpha$-hydroxylase gene and possibly also on turnover of cholesterol $7 \alpha$-hydroxylase messenger RNA (Unpublished observations). The mechanism appears independent of the negative feedback inhibition of cholesterol $7 \alpha$-hydroxylase by bile salts, which is thought to be mediated at a transcriptional level (11). In contrast to the rat, studies in human beings, including Kern's control group in this study, generally have shown little or no increase in bile acid synthesis in response to increased dietary cholesterol, although a single study by Lin and Conner (12) suggested that cholesterol feeding increased excretion of acidic sterols (bile salts) in feces. Recently, however, Mitchell and colleagues (13) found that the administration of lovastatin to human subjects leads to a 50\% drop in the rate of bile acid synthesis, consistent with previous findings in the rat.

How can we best put these data into perspective? Failure to detect up-regulation of bile acid synthesis in previous cholesterol feeding studies may indicate that this mechanism of adaptation in humans is operative only in the face of extreme changes in hepatic cholesterol, such as the extremely high cholesterol diet of the egg man or the acute inhibition of cholesterol synthesis by lovastatin. Alternatively, the capacity to regulate bile acid synthesis in response to changes in the supply of cholesterol may be a genetic variant and may account for the ability of some individuals to maintain normal serum cholesterol in the face of a high cholesterol diet.

Within the past $2 \mathrm{yr}$ the gene for rat cholesterol $7 \alpha$-hydroxylase has been cloned $(14,15)$. Intensive efforts currently are under way to dissect the $5^{\prime}$ regulatory region of this gene to explain the molecular mechanisms of the regulation of this important enzyme. In the future it may be possible to identify structural variations in the cholesterol $7 \alpha$-hydroxylase gene that are responsible for interindividual and interspecies differences in regulation of bile acid synthesis by cholesterol, as previously has been the case for the low-density lipoprotein receptor and for apolipoprotein E $(16,17,18)$.

Because of its role in atherosclerotic and coronary artery disease, serum cholesterol has been the subject of great attention. It must be kept in mind, however, that this is but one parameter of overall cholesterol homeostasis. Cholesterol metabolism is a complex process involving many branching metabolic pathways. These pathways are in turn regulated by a number of different molecules (apoproteins, receptors and enzymes) that are under both genetic and metabolic control. Given the complexity of the process, it is not surprising that individual responses to dietary cholesterol are so heterogeneous. Future efforts will need to address the genetic and physiological basis for these varied responses to more accurately identify those patients who would benefit from particular forms of dietary and pharmacological therapies. For now, we should keep in mind that some people, like the egg man, appear well adapted to the high cholesterol diet of Western society. To the extent that such individuals can be identified, it would be wrong to use our authority as counseling physicians to deny them the simple gustatory pleasures and nutritional benefits of the omelette, the custard and the soufflé.

MICHAEL P. JONES, M.D.
DOUGLAS M. HEUMAN, M.D.
Division of Gastroenterology
Medical College of Virginia
Richmond, VA 23298

\section{REFERENCES}

1. Goodman DS, et al. Report of the national cholesterol education program expert panel on detection, evaluation and treatment of high blood cholesterol in adults. Arch Intern Med 1988;148:36-69.

2. McNamara DJ, Kolb R, Parker TS, et al. Heterogeneity of cholesterol homeostasis in man: response to changes in dietary fat quality and cholesterol quantity. J Clin Invest 1987;79:1729-1739.

3. Turley SD, Dietschy JM. The metabolism and excretion of cholesterol by the liver. In: Arias IM, Popper H, Jakoby WB, Schachter D, Shafritz, eds. The liver: biology and pathobiology. 2nd ed. New York: Raven Press, 1988:617-641.

4. Arnesjo B, Nilsson A, Barrowman J, Borgstrom B. Intestinal digestion and absorption of cholesterol and lecithin in the human. Scand J Gastroenterol 1969;4:653-665.

5. Chow SL, Hollander D. Initial cholesterol uptake by everted sacs of rat small intestine: kinetic and thermodynamic aspects. Lipids 1978;13:239-245.

6. Thurhofer $H$, Hauser $H$. Uptake of cholesterol by small intestinal brush border membrane is protein mediated. Biochemistry 1990; 29:2142-2148. 
7. Vlahcevic ZR, Heuman DM, Hylemon PB. Regulation of bile acid synthesis. HEPATOLOGY 1991;13:590-600.

8. Raicht RF, Cohen BI, Shefer S, Mosbach EH. Sterol balance studies in the rat: effects of dietary cholesterol and $\beta$-sitosterol on sterol balance and rate-limiting enzymes of sterol metabolism. Biochim Biophys Acta 1975;388:374-384

9. Pandak WM, Heuman DM, Hylemon PB, Vlahcevic ZR. Regulation of bile acid synthesis. IV. Interrelationship between cholesterol and bile acid biosynthesis pathways. J Lipid Res 1990;31: 79-90.

10. Pandak WM, Vlahcevic ZR, Heuman DM, Hylemon PB. Regulation of bile acid synthesis. V. Inhibition of conversion of 7-dehydrocholesterol to cholesterol is associated with down regulation of cholesterol $7 \alpha$-hydroxylase activity and inhibition of bile acid synthesis. J Lipid Res 1991;31:2149-2158.

11. Pandak WM, Li YC, Chiang JYL, et. al. Regulation of cholesterol $7 \alpha$-hydroxylase mRNA and transcriptional activity by taurocholate and cholesterol in the chronic biliary diverted rat. $\mathbf{J}$ Biol Chem 1991;266:3416-3421

12. Lin DS, Connor WE. The long term effects of dietary cholesterol upon the plasma lipids, lipoproteins, cholesterol absorption and sterol balance in man: the demonstration of feedback inhibition of cholesterol biosynthesis and increased bile acid excretion. J Lipid Res 1980;21:1042-1052.

13. Mitchell JC, Stone BG, Logan GM, Duane WC. The role of cholesterol synthesis in regulation of bile acid synthesis and biliary cholesterol secretion in man [in press]. J Lipid Res 1991.

14. Jelinek DF, Russell DW. Structure of the rat gene encoding cholesterol 7 $\alpha$-hydroxylase. Biochem J 1990;29:7781-7785

15. Noshiro M, Nishimoto M, Okuda K. Rat liver cholesterol $7 \alpha$ hydroxylase: pretranslational regulation for circadian rhythm. J Biol Chem 1990;265:10036-10041.

16. Goldstein JL, Brown MS, Anderson RGW, et. al. Receptormediated endocytosis: concepts emerging from the LDL receptor system. Annu Rev Cell Biol 1985;1:1-39.

17. Kesaniemi YA, Ehnholm C, Miettinen TA. Intestinal cholesterol absorption efficiency in man is related to apoprotein $E$ phenotype. $J$ Clin Invest 1987;80:578-581.

18. Miettinin TA, Gylling H, Vanhanen $H$. Serum cholesterol response to dietary cholesterol and apoprotein $\mathrm{E}$ phenotype. Lancet $1988 ; 2: 1261$.

\section{EXCELLENT PROGNOSIS IN WLSONIAN CHRONIC ACTIVE HEPATITIS: NEW DATA OR AN ARTICLE OF FAITH?}

Schilsky ML, Scheinberg IH, Sternlieb I. Prognosis of Wilsonian chronic active hepatitis. Gastroenterology 1991;100:762-767.

\section{ABSTRACT}

Twenty of 320 patients with Wilson's disease initially presented with chemical and laboratory features of chronic active hepatitis, confirmed histologically in 17. When first seen, cirrhosis was present in all 20 and was complicated by ascites and/or jaundice in 11 . Within 1 week to 8 years of the onset of overt liver disease the diagnosis of Wilson's disease was established, and treatment with D-penicillamine was promptly initiated in 19 patients. One man refused treatment and died 4 months later. Treated patients received D-penicillamine or trientine for a total of 264 patient-years (median, 14 patient-years). Abnormal water retention, for which salt restriction and diuretics were added to penicillamine or trientine, disappeared in all but 1 of the patients so affected. Symptomatic improvement and virtually normal levels of serum albumin, bilirubin, aspartate ami- notransferase, and alanine aminotransferase followed within 1 year in the majority of subjects. One woman died after 9 months of treatment. Two patients, who became noncompliant with the therapeutic regimen after 9 and 17 years of successful pharmacological treatment, required liver transplants. These results indicate that the prognosis of specifically treated Wilsonian chronic active hepatitis is very good in spite of the presence of cirrhosis.

\section{COMMENTS}

Cirrhosis was part of the original description of Wilson's disease, and death from liver failure was probably recognized as early as $1916(1)$. With the advent of effective treatment with penicillamine, Wilsonian $\mathrm{CAH}$ has become the hepatological diagnosis that must not be missed! Just how good the prognosis can be is emphasized in this report. Although one patient died at 9 mo and 2 who became noncompliant with penicillamine treatment required liver transplantation, all 16 treated patients have done well for between 4 and $25 \mathrm{yr}$ despite the presence of cirrhosis at the time of diagnosis.

Twenty (6.25\%) of the 320 patients in the authors' series of Wilson's disease patients met the authors' criteria for histological and/or clinical features of CAH. In a previous study from the same group, it was reported that $42 \%$ of 151 symptomatic cases of Wilson's disease (which are presumably included in this study) had an initial hepatic manifestation (2). It seems clear, therefore, that the patients reported in this article represent a small subset of hepatic Wilson's disease and that the authors are drawing a clear distinction between Wilson's disease appearing as $\mathrm{CAH}$ and other forms of hepatic Wilson's disease, including fatty change, inactive cirrhosis and fulminant hepatitis. It would have been interesting to know how many of their total series fell into these other categories. Do the authors consider Wilsonian CAH to be part of a progression from an acute hepatitis-like injury to the characteristic inactive cirrhosis, or is it a distinct pathological entity? Likewise, is the feared fulminant presentation of Wilson's disease a distinct pathological entity or part of the spectrum of CAH? These questions are important because the reported excellent outcome of this particular type of Wilsonian chronic liver disease must, to some degree, depend on the extent to which it can be separated and distinguished from other varieties and from CAH of non-Wilsonian cause. For example, the experience of the Royal Free group (3), which had a similar series of patients with Wilson's disease appearing as $\mathrm{CAH}$, was much less encouraging. They noted that "despite D-penicillamine therapy . . . 4 patients died within three weeks of diagnosis from fulminant hepatic failure... (and) a further 5 patients died within 2 years. ..."

The diagnosis of Wilson's disease in the patients described by Schilsky, Scheinberg and Sternlieb, as would be expected in a paper from this group, appears to be beyond question. Nonetheless, we are told that several other causes of $\mathrm{CAH}$, "including hepatitis type $\mathrm{B}$, 